ANNALES

POLONICI MATHEMATICI

$98.2(2010)$

\title{
A stochastic symbiosis model with degenerate diffusion process
}

\author{
by URSZULA SKWARA (Lublin)
}

\begin{abstract}
We present a model of symbiosis given by a system of stochastic differential equations. We consider a situation when the same factor influences both populations or only one population is stochastically perturbed. We analyse the long-time behaviour of the solutions and prove the asymptoptic stability of the system.
\end{abstract}

1. Introduction. The aim of this paper is to study the following system of stochastic differential equations:

$$
\begin{aligned}
& d X(t)=\left(\left(a_{1}+b_{1} Y(t)-c_{1} X(t)\right) d t+\rho_{1} d W(t)\right) X(t), \\
& d Y(t)=\left(\left(a_{2}+b_{2} X(t)-c_{2} Y(t)\right) d t+\rho_{2} d W(t)\right) Y(t) .
\end{aligned}
$$

This is a stochastic version of the deterministic symbiosis model [7] (Gause and Witt, 1935)

$$
x^{\prime}=\left(a_{1}+b_{1} y-c_{1} x\right) x, \quad y^{\prime}=\left(a_{2}+b_{2} x-c_{2} y\right) y .
$$

where the functions $x(t), y(t)$ represent, respectively, the size of the first and the second population at time $t$. We assume that $a_{i}, b_{i}, c_{i}(i=1,2)$ are positive constants. The coefficients $a_{i}(i=1,2)$ are ideal growth rates, $b_{i}$ $(i=1,2)$ are symbiosis rates, $c_{i}(i=1,2)$ are death rates.

In the model described by (1) and (2) the stochastic processes $X(t)$, $Y(t)$ represent, respectively, the first and the second population, $W(t)$ is a one-dimensional standard Wiener process, the constants $a_{i}, b_{i}, c_{i}(i=1,2)$, as in the deterministic model, are positive, and $\rho_{i}(i=1,2)$ are diffusion coefficients. We assume that fluctuations of the environment randomly change the reproduction rates of the populations and the random noise is proportional to the number of individuals. We consider two kinds of stochastic perturbations:

(i) strongly correlated, i.e. $\rho_{1}>0, \rho_{2}>0$,

2010 Mathematics Subject Classification: 47D07, 35K15, 60J60, 60H10, 92D25.

Key words and phrases: symbiosis model, diffusion process, Markov semigroups, asymptotic stability. 
(ii) only one population is stochastically perturbed; by symmetry, we assume that the second population is perturbed, i.e. $\rho_{1}=0, \rho_{2}>0$.

Case (i) corresponds to the situation when the same factor (like weather conditions) affects both populations.

We consider system (1), (2) on the assumption that $b_{1} b_{2}<c_{1} c_{2}$, because in the deterministic model (3) we observe that the sizes of both populations go to infinity if $b_{1} b_{2} \geq c_{1} c_{2}$. The inequality $b_{1} b_{2}<c_{1} c_{2}$ has an interesting biological interpretation, namely it means that the symbiosis coefficients are smaller than the death rates. The goal of this paper is to study the long-time behaviour of the solutions of system (1), (2).

We prove that the probability distributions of the process $(X(t), Y(t))$ are absolutely continuous with respect to Lebesgue measure. Let $U(x, y, t)$ be the density of the distribution of $(X(t), Y(t))$. We give a sufficient and a necessary condition for the asymptotic stability of system (1), (2), i.e. the convergence of $U(x, y, t)$ to an invariant density $U_{*}(x, y)$. We also find the support of $U_{*}(x, y)$. If the system is not asymptotically stable, we prove that $\lim _{t \rightarrow \infty} Y(t)=0$ a.e. We also show that in this case either $\lim _{t \rightarrow \infty} X(t)=0$ a.e., or the probability distribution of the process $X(t)$ converges weakly to some probability measure.

A model of symbiosis in which stochastic perturbations are weakly correlated was considered in [28. System (1), (2) has similar properties, but methods of the proof are more complicated. In the same way as in [28] we prove the existence, uniqueness, positivity and non-extinction property of the solutions. But it is more difficult to obtain the asymptotic stability, because the Fokker-Planck equation corresponding to system (1), (2) is of a degenerate type. We use Hörmander's theorem ([2, [9], [15], [16], [19]) in order to show that a semigroup connected with the Fokker-Planck is integral and has a continuous kernel. Using support theorems ([1, [3, [13, [29]) we find a set $E$ on which the kernel is positive. Next we prove that $E$ is an "attractor". Then we apply some facts concerning integral Markov semigroups ([20], [21], 24] and [27]) to show that the semigroup connected with the Fokker-Planck equation satisfies the "Foguel alternative", i.e. it is either asymptotically stable or "sweeping". Finally, we find a Khasminskiu function which excludes "sweeping" and in this way we obtain the asymptotic stability.

A similar technique was applied to study properties of a stochastic preypredator model [25]. It should be noted that in [28] the proof of the asymptotic stability is much easier because the semigroup connected with the Fokker-Planck equation is integral and has a continuous and strictly positive kernel. Therefore, in order to obtain the asymptotic stability it is sufficient to construct a Khasminskil function. When system (1), (2) is not asymptotically stable we prove similar results to those in [28] using the same methods. 
In particular, we show that in this case one of the populations becomes extinct.

2. Mathematical results and their interpretation. In this section we formulate the main results of this paper.

THEOREM 1. If $b_{1} b_{2}<c_{1} c_{2}$ then for any initial condition $(X(0), Y(0)) \in$ $\mathbb{R}_{+}^{2}$ there exists a unique solution $(X(t), Y(t))$ of system (1), (2) for $t \geq 0$ and the solution remains in $\mathbb{R}_{+}^{2}$ with probability 1 , i.e. $(X(t), Y(t)) \in \mathbb{R}_{+}^{2}$ for all $t \geq 0$ almost surely.

The proof of this theorem is identical to the case of weakly correlated perturbations (see Theorem 2 in 28]).

The asymptotic behaviour of system (1), (2) depends on the constants $b_{1}, b_{2}, c_{1}, c_{2}, \rho_{1}, \rho_{2}, \tilde{a}_{1}=a_{1}-\rho_{1}^{2} / 2, \tilde{a}_{2}=a_{2}-\rho_{2}^{2} / 2$.

THEOREM 2. Let $b_{1} b_{2}<c_{1} c_{2}$. If $(X(t), Y(t))$ is a solution of system (1), (2), then for every $t>0$ the distribution of $(X(t), Y(t))$ has a density $U(t, x, y)$.

(I) Let $\tilde{a}_{1}, \tilde{a}_{2}>0$. In case (i), assume that $\tilde{a}_{1} \neq \tilde{a}_{2}$ or $\rho_{1} \neq \rho_{2}$. Then there exists a unique invariant density $U_{*}(x, y)$ such that

$$
\lim _{t \rightarrow \infty} \iint_{\mathbb{R}_{+}^{2}}\left|U(x, y, t)-U_{*}(x, y)\right| d x d y=0 .
$$

(II) If $\tilde{a}_{1}, \tilde{a}_{2}<0$ then

$$
\lim _{t \rightarrow \infty} X(t)=0 \text { a.e. and } \lim _{t \rightarrow \infty} Y(t)=0 \text { a.e. }
$$

(III) Let $\tilde{a}_{1}>0, \tilde{a}_{2}<0$ and $\tilde{a}_{1} b_{2}+\tilde{a}_{2} c_{1}>0$. Then there exists a unique invariant density $U_{*}(x, y)$ such that

$$
\lim _{t \rightarrow \infty} \iint_{\mathbb{R}_{+}^{2}}\left|U(x, y, t)-U_{*}(x, y)\right| d x d y=0 .
$$

(IV) Let $\tilde{a}_{1}>0, \tilde{a}_{2}<0$ and $\tilde{a}_{1} b_{2}+\tilde{a}_{2} c_{1}<0$. Then in case (i) we have $\lim _{t \rightarrow \infty} Y(t)=0$ a.e. and the distribution of the process $X(t)$ converges weakly to the measure with density

$$
f_{*}(x)=C x^{2 \tilde{a}_{1} / \rho_{1}^{2}-1} \exp \left(-2 c_{1} x / \rho_{1}^{2}\right),
$$

where $C=\left(2 c_{1} / \rho_{1}{ }^{2}\right)^{2 \tilde{a}_{1} / \rho_{1}{ }^{2}} / \Gamma\left(2 \tilde{a}_{1} / \rho_{1}{ }^{2}\right)$, while in case (ii) we have $\lim _{t \rightarrow \infty} Y(t)=0$ a.e. and $\lim _{t \rightarrow \infty} X(t)=a_{1} / c_{1}$ a.e.

In cases (I) and (III) the support of the invariant density $U_{*}$ depends on the coefficients $\rho_{1}, \rho_{2}, \tilde{a}_{1}, \tilde{a}_{2}$. By the support of a measurable function $f$ we simply mean the set

$$
\operatorname{supp} f=\{(x, y) \in X: f(x, y) \neq 0\} .
$$

We have the following result. 
TheOREM 3. Let $b_{1} b_{2}<c_{1} c_{2}$. If both populations are stochastically perturbed then in case (I) we have four subcases:

(a $\left.\mathrm{a}_{1}\right)$ If $\rho_{2}>\rho_{1}$ and $\tilde{a}_{2} \rho_{1}>\tilde{a}_{1} \rho_{2}$ then $\operatorname{supp} U_{*}=\mathbb{R}_{+}^{2}$.

$\left(\mathrm{b}_{1}\right)$ If $\rho_{2} \leq \rho_{1}$ and $\tilde{a}_{2} \rho_{1}>\tilde{a}_{1} \rho_{2}$ then there exists a constant $C_{1}$ such that

$$
\operatorname{supp} U_{*}=\left\{(x, y) \in \mathbb{R}_{+}^{2}: x<C_{1} y^{\rho_{1} / \rho_{2}}\right\} .
$$

$\left(\mathrm{c}_{1}\right)$ If $\rho_{2}<\rho_{1}$ and $\tilde{a}_{2} \rho_{1}<\tilde{a}_{1} \rho_{2}$ then $\operatorname{supp} U_{*}=\mathbb{R}_{+}^{2}$.

$\left(\mathrm{d}_{1}\right)$ If $\rho_{2} \geq \rho_{1}$ and $\tilde{a}_{2} \rho_{1}<\tilde{a}_{1} \rho_{2}$ then there exists a constant $C_{1}$ such that

$$
\operatorname{supp} U_{*}=\left\{(x, y) \in \mathbb{R}_{+}^{2}: y<C_{1} x^{\rho_{2} / \rho_{1}}\right\} .
$$

If both populations are stochastically perturbed then in case (III) we have two subcases:

(a) If $\rho_{2}<\rho_{1}$ then $\operatorname{supp} U_{*}=\mathbb{R}_{+}^{2}$.

$\left(\mathrm{b}_{3}\right)$ If $\rho_{2} \geq \rho_{1}$ then there exists a constant $C_{1}$ such that

$$
\operatorname{supp} U_{*}=\left\{(x, y) \in \mathbb{R}_{+}^{2}: y<C_{1} x^{\rho_{2} / \rho_{1}}\right\} .
$$

If the second population is stochastically perturbed then

$$
\operatorname{supp} U_{*}=\left(a_{1} / c_{1}, \infty\right) \times \mathbb{R}_{+} .
$$

REMARK 1. As equations (1), (2) are symmetrical we omit some cases in the statement of Theorem 2. For example, we do not take into account the case $\tilde{a}_{1}<0, \tilde{a}_{2}>0, \tilde{a}_{2} b_{1}+\tilde{a}_{1} c_{2}>0$, because it is symmetrical to (III), and the case $\tilde{a}_{1}<0, \tilde{a}_{2}>0, \tilde{a}_{2} b_{1}+\tilde{a}_{1} c_{2}<0$, symmetrical to (IV).

REMARK 2. In order to explain the role of the coefficients $\tilde{a}_{1}, \tilde{a}_{2}$ we consider the following equation of population growth:

$$
d N(t)=(a d t+\rho d W(t)) N(t),
$$

where $a>0$ is the growth rate, $\rho>0$ is a diffusion coefficient, $W(t)$ is a one-dimensional standard Wiener process, and $N(t)$ is a real stochastic process. The solution of this equation is of the form

$$
N(t)=N(0) \exp \{\tilde{a} t+\rho W(t)\},
$$

where $\tilde{a}=a-\frac{1}{2} \rho^{2}$. The constant $\tilde{a}$ is called the new growth rate or for short the growth rate of the population. It is easy to check that if $\tilde{a}>0$ then $\lim _{t \rightarrow \infty} N(t)=\infty$ a.e., and if $\tilde{a}<0$ then $\lim _{t \rightarrow \infty} N(t)=0$ a.e. In other words, the positivity of the new growth rate means that the stochastic perturbation is small and therefore the size of the population goes to infinity as in the deterministic case. The negativity of the new growth rate means that the stochastic perturbation is too large and therefore the population dies out. Even though we consider more complicated equations, we will call the coefficients $\tilde{a}_{1}, \tilde{a}_{2}$ the new growth rates or briefly growth rates of these populations. 
Remark 3. Theorems 2 and 3 have an interesting biological interpretation.

From Theorem 2 it follows that if the new growth rate of the population is positive then this population survives. Otherwise, if the new growth rate is negative then the population may die out. The most interesting is case (III), because we observe a positive influence of symbiosis. Namely, from (III) it follows that if the new growth rate for one population is positive and for the other it is negative, but benefits of symbiosis for the second population are large, then both populations survive.

Theorem 3 provides us with information about the support of the invariant density $U_{*}(x, y)$. If we have a nondegenerate diffusion process as in [28] then the support is the whole space, but in our case it may be some proper subset. Especially interesting are formulas (6)-(9). From Theorem 2 it follows that the distribution of the system $(X(t), \bar{Y}(t))$ can converge to an invariant distribution with some density $U_{*}$. Let a pair of variables $(X, Y)$ have the density distribution $U_{*}$. Then, for example, from (6) we obtain $X<C_{1} Y^{\rho_{1} / \rho_{2}}$. This means that the second population controls the size of the first.

3. Markov semigroups. In this section we give some facts concerning Markov semigroups.

Let $(X, \Sigma, m)$ be a $\sigma$-finite measure space. Denote by $D$ the subset of $L^{1}=L^{1}(X, \Sigma, m)$ which consists of all densities, i.e.

$$
D=\left\{f \in L^{1}: f \geq 0,\|f\|=1\right\} .
$$

A linear mapping $P: L^{1} \rightarrow L^{1}$ is called a Markov operator if $P(D) \subset D$.

The Markov operator $P$ is called an integral operator if there exists a measurable function $k: X \times X \rightarrow[0, \infty)$ such that

$$
P f(x)=\int_{X} k(x, y) f(y) m(d y)
$$

for every density $f$. The function $k$ is called the kernel of the operator $P$. One can check that from the condition $P(D) \subset D$ it follows that

$$
\int_{X} k(x, y) m(d x)=1
$$

for almost all $y \in X$.

A family $\{P(t)\}_{t \geq 0}$ of Markov operators which satisfies:

(a) $P(0)=\mathrm{Id}$,

(b) $P(t+s)=P(t) P(s)$ for $s, t \geq 0$,

(c) for each $f \in L^{1}$ the function $t \mapsto P(t) f$ is continuous with respect to the $L^{1}$ norm 
is called a Markov semigroup. A Markov semigroup $\{P(t)\}_{t \geq 0}$ is called integral if for each $t>0$, the operator $P(t)$ is an integral Markov operator.

We also need two definitions concerning the asymptotic behaviour of a Markov semigroup. A density $f_{*}$ is called invariant if $P(t) f_{*}=f_{*}$ for each $t>0$. The Markov semigroup $\{P(t)\}_{t \geq 0}$ is called asymptotically stable if there is an invariant density $f_{*}$ such that

$$
\lim _{t \rightarrow \infty}\left\|P(t) f-f_{*}\right\|=0 \quad \text { for } f \in D .
$$

A Markov semigroup $\{P(t)\}_{t \geq 0}$ is called sweeping with respect to a set $A \in \Sigma$ if for every $f \in D$,

$$
\lim _{t \rightarrow \infty} \int_{A} P(t) f(x) m(d x)=0 .
$$

Theorem $4([25])$. Let $X$ be a metric space and $\Sigma$ be the $\sigma$-algebra of Borel sets. Let $\{P(t)\}_{t \geq 0}$ be an integral Markov semigroup with a continuous kernel $k(t, x, y)$ for $t>0$, which satisfies (12) for all $y \in X$. Assume that for every $f \in D$,

$$
\int_{0}^{\infty} P(t) f d t>0 \quad \text { a.e. }
$$

Then this semigroup is asymptotically stable or sweeping with respect to compact sets.

The property that a Markov semigroup $\{P(t)\}_{t \geq 0}$ is asymptotically stable or sweeping from a sufficiently large family of sets (e.g. from all compact sets) is called the Foguel alternative.

4. Properties of trajectories. Now instead of system (1), (2) we consider a simpler system in which the diffusion coefficients are constant. We substitute $X(t)=e^{\xi(t)}, Y(t)=e^{\eta(t)}$. Using the Itô formula we obtain

$$
\begin{aligned}
& d \xi(t)=\left(\tilde{a}_{1}+b_{1} e^{\eta(t)}-c_{1} e^{\xi(t)}\right) d t+\rho_{1} d W(t), \\
& d \eta(t)=\left(\tilde{a}_{2}+b_{2} e^{\xi(t)}-c_{2} e^{\eta(t)}\right) d t+\rho_{2} d W(t) .
\end{aligned}
$$

The proof of parts (II) and (IV) of Theorem 2 is similar to the case with weakly correlated perturbations (see [28]). Some differences are in part (IV) when only the second population is stochastically perturbed and therefore we prove the following result.

LEMma 1. Let $b_{1} b_{2}<c_{1} c_{2}, \rho_{1}=0, \rho_{2}>0$. If $\tilde{a}_{2}<0$ and $a_{1} b_{2}+\tilde{a}_{2} c_{1}<0$ then

$$
\lim _{t \rightarrow \infty} \xi(t)=\log \left(a_{1} / c_{1}\right) \quad \text { a.e. and } \lim _{t \rightarrow \infty} \eta(t)=-\infty \quad \text { a.e. }
$$

Before the proof we recall an obvious theorem on differential equations. 
Theorem 5. Let $P \subset \mathbb{R}$ be an open interval and $h: P \times[0, \infty) \rightarrow \mathbb{R}$ be a differentiable function. Suppose that for every compact interval $P_{0} \subset P$, $h(x, t)$ converges uniformly to $g(x)$ in $P_{0}$ as $t \rightarrow \infty$. Moreover, assume that the following conditions are satisfied:

(a) there exists $\beta \in P_{0}$ such that $g(x)>0$ for $x<\beta$ and $g(x)<0$ for $x>\beta$,

(b) there exist $a, b \in P_{0}$ with $a<\beta<b$ such that $h(x, t)>0$ for $x<a$, $t \in[0, \infty)$, and $h(x, t)<0$ for $x>b, t \in[0, \infty)$.

Then there exists a solution $x(t)$ of the equation

$$
x^{\prime}(t)=h(x, t)
$$

for all $t>0$, which satisfies the condition

$$
\lim _{t \rightarrow \infty} x(t)=\beta .
$$

Proof of Lemma 5. First we show that $\lim _{t \rightarrow \infty} \eta(t)=-\infty$ a.e. Multiplying (15) by $b_{2}$ and (16) by $c_{1}$ and adding these equations we have

$$
d\left(b_{2} \xi(t)+c_{1} \eta(t)\right)=\left(a_{1} b_{2}+\tilde{a}_{2} c_{1}+\left(b_{1} b_{2}-c_{1} c_{2}\right) e^{\eta(t)}\right) d t+c_{1} \rho_{2} d W(t) .
$$

Since $b_{1} b_{2}<c_{1} c_{2}$, from the comparison theorem [8, Lemma 4, p. 120] we have

$$
d\left(b_{2} \xi(t)+c_{1} \eta(t)\right) \leq\left(a_{1} b_{2}+\tilde{a}_{2} c_{1}\right) d t+c_{1} \rho_{2} d W(t) .
$$

Consequently,

$$
\lim _{t \rightarrow \infty}\left(b_{2} \xi(t)+c_{1} \eta(t)\right)=-\infty \quad \text { a.e. }
$$

Thus for arbitrarily small $\varepsilon>0$ there exist $t_{0}$ and a set $\Omega_{\varepsilon}$ such that $\operatorname{Prob}\left(\Omega_{\varepsilon}\right)>1-\varepsilon$ and $\xi(t)<-\left(c_{1} / b_{2}\right) \eta(t)$ for $t \geq t_{0}$ and $\omega \in \Omega_{\varepsilon}$. It follows that

$$
d \eta(t) \leq\left(\tilde{a}_{2}+b_{2} e^{-\left(c_{1} / b_{2}\right) \eta(t)}\right) d t+\rho_{2} d W(t) .
$$

Consider the equation

$$
d \bar{\eta}(t)=\left(\tilde{a}_{2} / 2+b_{2} e^{-\left(c_{1} / b_{2}\right) \bar{\eta}(t)}\right) d t+\rho_{2} d W(t) .
$$

The Fokker-Planck equation corresponding to 18 has a stationary density

$$
f_{*}(x)=C \exp \left(\frac{2}{\rho_{2}^{2}}\left(\frac{\tilde{a}_{2}}{2} x-\frac{b_{2}^{2}}{c_{1}} e^{-\left(c_{1} / b_{2}\right) x}\right)\right),
$$

where $C$ is some constant. From the ergodic theorem [8, Theorem 2, p. 141] it follows that

$$
\lim _{t \rightarrow \infty} \frac{1}{t} \int_{0}^{t} e^{-\left(c_{1} / b_{2}\right) \bar{\eta}(s)} d s=\int_{-\infty}^{\infty} f_{*}(x) e^{-\left(c_{1} / b_{2}\right) x} d x
$$


Since $f_{*}^{\prime}(x)=\left(2 / \rho_{2}^{2}\right)\left(\tilde{a}_{2} / 2+b_{2} e^{-\left(c_{1} / b_{2}\right) x}\right) f_{*}(x)$ we have

$$
\int_{-\infty}^{\infty} f_{*}(x) e^{-\left(c_{1} / b_{2}\right) x} d x=-\frac{\tilde{a}_{2}}{2 b_{2}}>0 .
$$

From (19), 20) we obtain

$$
\lim _{t \rightarrow \infty} \frac{1}{t} \int_{0}^{t} e^{-\left(c_{1} / b_{2}\right) \bar{\eta}(s)} d s=-\frac{\tilde{a}_{2}}{2 b_{2}} .
$$

From (17) we have

$$
\eta(t) \leq \bar{\eta}(t)+\frac{\tilde{a}_{2}}{2} t+C_{1}
$$

where $\bar{\eta}(t)$ is a solution of equation (18) and $C_{1}$ is some constant. Therefore,

$$
\eta(t) \leq \tilde{a}_{2} t+b_{2} \int_{0}^{t} e^{-\left(c_{1} / b_{2}\right) \bar{\eta}(s)} d s+\rho_{2} W(t)+C_{1} .
$$

From this, 21), and $\lim _{t \rightarrow \infty} W(t) / t=0$ we obtain

$$
\limsup _{t \rightarrow \infty} \frac{\eta(t)}{t} \leq \frac{\tilde{a}_{2}}{2}<0,
$$

and consequently $\lim _{t \rightarrow \infty} \eta(t)=-\infty$ a.e. The process $\xi(t)$ satisfies the equation

$$
d \xi(t)=\left(a_{1}+b_{1} e^{\eta(t)}-c_{1} e^{\xi(t)}\right) d t .
$$

If $h(x, t)=a_{1}+b_{1} e^{\eta(t)}-c_{1} e^{x}, g(x)=a_{1}-c_{1} e^{x}$ then from Theorem 5 we obtain

$$
\lim _{t \rightarrow \infty} \xi(t)=\log \left(a_{1} / c_{1}\right) \quad \text { a.e. }
$$

5. Asymptotic stability. Let $(\xi(t), \eta(t))$ be a solution of (15), (16) such that the distribution of $(\xi(0), \eta(0))$ is absolutely continuous with density $v(x, y)$. Then the random variable $(\xi(t), \eta(t))$ has the density $u(x, y, t)$ and $u$ satisfies the Fokker-Planck equation

$$
\frac{\partial u}{\partial t}=\frac{1}{2} \rho_{1}^{2} \frac{\partial^{2} u}{\partial x^{2}}+\rho_{1} \rho_{2} \frac{\partial^{2} u}{\partial x \partial y}+\frac{1}{2} \rho_{2}^{2} \frac{\partial^{2} u}{\partial y^{2}}-\frac{\partial\left(f_{1} u\right)}{\partial x}-\frac{\partial\left(f_{2} u\right)}{\partial y},
$$

where $f_{1}(x, y)=\tilde{a}_{1}+b_{1} e^{y}-c_{1} e^{x}, f_{2}(x, y)=\tilde{a}_{2}+b_{2} e^{x}-c_{2} e^{y}$.

Now we introduce a Markov semigroup connected with the FokkerPlanck equation 221. Let $X=\mathbb{R}^{2}, \Sigma$ be the $\sigma$-algebra of Borel subsets of $X$, and $m$ be the Lebesgue measure on $(X, \Sigma)$. Let $P(t) v(x, y)=u(x, y, t)$ for $v \in D$. Since the operator $P(t)$ is a contraction on $D$, it can be extended to a contraction on $L^{1}\left(\mathbb{R}^{2}, \Sigma, m\right)$. Thus the operators $\{P(t)\}_{t \geq 0}$ form a Markov semigroup. Let $\mathcal{A}$ be the infinitesimal generator of the semigroup $\{P(t)\}_{t \geq 0}$, 
i.e.

$$
\mathcal{A} v=\frac{1}{2} \rho_{1}^{2} \frac{\partial^{2} v}{\partial x^{2}}+\rho_{1} \rho_{2} \frac{\partial^{2} v}{\partial x \partial y}+\frac{1}{2} \rho_{2}^{2} \frac{\partial^{2} v}{\partial y^{2}}-\frac{\partial\left(f_{1} v\right)}{\partial x}-\frac{\partial\left(f_{2} v\right)}{\partial y} .
$$

The adjoint operator of $\mathcal{A}$ is of the form

$$
\mathcal{A}^{*} v=\frac{1}{2} \rho_{1}^{2} \frac{\partial^{2} v}{\partial x^{2}}+\rho_{1} \rho_{2} \frac{\partial^{2} v}{\partial x \partial y}+\frac{1}{2} \rho_{2}^{2} \frac{\partial^{2} v}{\partial y^{2}}+f_{1} \frac{\partial v}{\partial x}+f_{2} \frac{\partial v}{\partial y} .
$$

Let $\mathcal{P}(t, x, y, A)$ be the transition probability function for the diffusion process $(\xi(t), \eta(t))$, i.e. $\mathcal{P}(t, x, y, A)=\operatorname{Prob}((\xi(t), \eta(t)) \in A)$ and $(\xi(t), \eta(t))$ is a solution of system (15), 16 with the initial condition $(\xi(0), \eta(0))=$ $(x, y)$.

The aim of this section is to prove the asymptotic stability of the semigroup $\{P(t)\}_{t \geq 0}$, because it implies the convergence in $L^{1}$ of the densities $u(x, y, t)$ of the process $(\xi(t), \eta(t))$ to the invariant density. Therefore instead of proving parts (I) and (III) of Theorem 2 we show the asymptotic stability of this semigroup.

If stochastic perturbations are weakly correlated then the semigroup connected with the Fokker-Planck equation is an integral Markov semigroup with a continuous and strictly positive kernel. In our case we use Hörmander's theorem on the existence of smooth densities of the transition probability function for degenerate diffusion processes in order to prove that this semgroup is integral and has a continuous kernel. Let us recall this theorem now.

Consider the Stratonovitch stochastic differential equation

$$
d X(t)=\sigma(X(t)) \circ d W(t)+\sigma_{0}(X(t)) d t,
$$

where $W(t)$ is an $m$-dimensional Brownian motion, $\sigma(x)=\left[\sigma_{j}^{i}(x)\right]$ is a $d \times m$ matrix and $\sigma_{0}(x)$ is a vector in $\mathbb{R}^{d}$ with components $\sigma_{0}^{i}(x)$ for every $x \in \mathbb{R}^{d}$. Let $\sigma_{j}(x)(j=0, \ldots, m)$ be a vector in $\mathbb{R}^{d}$ with components $\sigma_{j}^{i}(x)$ for every $x \in \mathbb{R}^{d}$. If $a(x)$ and $b(x)$ are two vector fields on $\mathbb{R}^{d}$ then their Lie bracket $[a, b]$ is the vector field on $\mathbb{R}^{d}$ given by

$$
[a, b]_{j}(x)=\sum_{k=1}^{d}\left(a_{k} \frac{\partial b_{j}}{\partial x_{k}}(x)-b_{k} \frac{\partial a_{j}}{\partial x_{k}}(x)\right) .
$$

TheOREM 6 (Hörmander). If for every $x \in \mathbb{R}^{d}$ the vectors

$$
\sigma_{1}(x), \ldots, \sigma_{m}(x),\left[\sigma_{i}, \sigma_{j}\right](x)_{0 \leq i, j \leq m},\left[\sigma_{i},\left[\sigma_{j}, \sigma_{k}\right]\right](x)_{0 \leq i, j, k \leq m}, \ldots
$$

span the space $\mathbb{R}^{d}$ then the transition probability function $\mathcal{P}(t, x, A)$ has a density $k(t, y, x)$ and $k \in C^{\infty}\left((0, \infty) \times \mathbb{R}^{d} \times \mathbb{R}^{d}\right)$.

LEMMA 2. Let $\rho_{1}, \rho_{2}>0$. If $\rho_{1} \neq \rho_{2}$ or $\tilde{a}_{1} \neq \tilde{a}_{2}$ then $\{P(t)\}_{t \geq 0}$ is an integral Markov semigroup with a continuous kernel $k$. 
Proof. Let $\sigma_{0}(\xi, \eta)=\left(\tilde{a}_{1}+b_{1} e^{\eta}-c_{1} e^{\xi}, \tilde{a}_{2}+b_{2} e^{\xi}-c_{2} e^{\eta}\right)$ and $\sigma_{1}(\xi, \eta)=$ $\left(\rho_{1}, \rho_{2}\right)$. Then

$$
\begin{aligned}
{\left[\sigma_{0}, \sigma_{1}\right](\xi, \eta) } & =\left(c_{1} \rho_{1} e^{\xi}-b_{1} \rho_{2} e^{\eta}, c_{2} \rho_{2} e^{\eta}-b_{2} \rho_{1} e^{\xi}\right), \\
{\left[\sigma_{1},\left[\sigma_{0}, \sigma_{1}\right]\right](\xi, \eta) } & =\left(c_{1} \rho_{1}^{2} e^{\xi}-b_{1} \rho_{2}^{2} e^{\eta}, c_{2} \rho_{2}^{2} e^{\eta}-b_{2} \rho_{1}^{2} e^{\xi}\right) .
\end{aligned}
$$

If $\rho_{1} \neq \rho_{2}$ then for every $(\xi, \eta) \in \mathbb{R}^{2}$ the vectors $\sigma_{1}(\xi, \eta),\left[\sigma_{0}, \sigma_{1}\right](\xi, \eta)$, $\left[\sigma_{1},\left[\sigma_{0}, \sigma_{1}\right]\right](\xi, \eta)$ span $\mathbb{R}^{2}$. If $\rho_{1}=\rho_{2}$ and $\tilde{a}_{1} \neq \tilde{a}_{2}$ then the vectors

$$
\begin{gathered}
\sigma_{1}(\xi, \eta)=\left(\rho_{1}, \rho_{1}\right), \quad\left[\sigma_{0}, \sigma_{1}\right](\xi, \eta)=\rho_{1}\left(c_{1} e^{\xi}-b_{1} e^{\eta}, c_{2} e^{\eta}-b_{2} e^{\xi}\right), \\
{\left[\sigma_{0},\left[\sigma_{0}, \sigma_{1}\right]\right](\xi, \eta)=\rho_{1}\left(\tilde{a}_{1} c_{1} e^{\xi}-\tilde{a}_{2} b_{1} e^{\eta}, \tilde{a}_{2} c_{2} e^{\eta}-\tilde{a}_{1} b_{2} e^{\xi}\right)}
\end{gathered}
$$

span $\mathbb{R}^{2}$ for every $(\xi, \eta) \in \mathbb{R}^{2}$. From Hörmander's theorem it follows that $\mathcal{P}\left(t, x_{0}, y_{0}, \cdot\right)$ has a density $k\left(t, x, y ; x_{0}, y_{0}\right)$ and $k \in C^{\infty}\left((0, \infty) \times \mathbb{R}^{2} \times \mathbb{R}^{2}\right)$. Thus

$$
P(t) f(x, y)=\int_{-\infty}^{\infty} \int_{-\infty}^{\infty} k(t, x, y ; u, v) f(u, v) d u d v .
$$

This means that $\{P(t)\}_{t \geq 0}$ is an integral Markov semigroup.

REMARK 4. Let $\rho_{1}, \rho_{2}>0$. If $\rho_{1}=\rho_{2}$ and $\tilde{a}_{1}=\tilde{a}_{2}$ then considering a solution of system (15), (16) with initial conditions $\xi(0)=x_{0}, \eta(0)=y_{0}$ such that

$$
y_{0}=x_{0}+\log \frac{b_{2}+c_{1}}{b_{1}+c_{2}}
$$

we obtain

$$
\eta(t)=\xi(t)+\log \frac{b_{2}+c_{1}}{b_{1}+c_{2}}
$$

and the transition density $k\left(t, x, y ; x_{0}, y_{0}\right)$ does not exist.

Lemma 3. If $\rho_{1}=0, \rho_{2}>0$ then $\{P(t)\}_{t \geq 0}$ is an integral Markov semigroup with a continuous kernel $k$.

Proof. Let $\sigma_{0}(\xi, \eta)=\left(a_{1}+b_{1} e^{\eta}-c_{1} e^{\xi}, \tilde{a}_{2}+b_{2} e^{\xi}-c_{2} e^{\eta}\right)$ and $\sigma_{1}(\xi, \eta)=$ $\left(0, \rho_{2}\right)$. Since $\left[\sigma_{0}, \sigma_{1}\right](\xi, \eta)=\rho_{2}\left(-b_{1} e^{\eta}, c_{2} e^{\eta}\right)$, for every $(\xi, \eta) \in \mathbb{R}^{2}$ the vectors $\sigma_{1}(\xi, \eta)$ and $\left[\sigma_{0}, \sigma_{1}\right](\xi, \eta)$ span the space $\mathbb{R}^{2}$. Using the same arguments as in the proof of the previous lemma we conclude that $\{P(t)\}_{t \geq 0}$ is an integral Markov semigroup with a continuous kernel.

Now we find the support of the kernel $k$. If a diffusion process is nondegenerate then the support is the whole space, but in our case we use support theorems in order to find the set in which the kernel is positive. Now we describe the method based on support theorems [1, 3, 29]. Fix a point $\left(x_{0}, y_{0}\right) \in \mathbb{R}^{2}$ and a continuous function $\phi:[0, T] \rightarrow \mathbb{R}$. Consider the 
system

$$
\begin{aligned}
x_{\phi}^{\prime}(t)=\rho_{1} \phi(t)+f_{1}\left(x_{\phi}(t), y_{\phi}(t)\right), & x_{\phi}(0)=x_{0}, \\
y_{\phi}^{\prime}(t)=\rho_{2} \phi(t)+f_{2}\left(x_{\phi}(t), y_{\phi}(t),\right. & y_{\phi}(0)=y_{0},
\end{aligned}
$$

where $f_{1}(x, y)=\tilde{a}_{1}+b_{1} e^{y}-c_{1} e^{x}, f_{2}(x, y)=\tilde{a}_{2}+b_{2} e^{x}-c_{2} e^{y}, \rho_{1}=0$ in case (ii). Let $D_{x_{0}, y_{0} ; \phi}$ be the Fréchet derivative of the function

$$
h \mapsto \mathbf{x}_{\phi+h}(T) \quad \text { with } \quad \mathbf{x}_{\phi+h}=\left[\begin{array}{l}
x_{\phi+h} \\
y_{\phi+h}
\end{array}\right] .
$$

If for some $\phi$ the derivative $D_{x_{0}, y_{0} ; \phi}$ has rank 2 then $k\left(T, x, y ; x_{0}, y_{0}\right)>0$ for $x=x_{\phi}(T)$ and $y=y_{\phi}(T)$. The derivative $D_{x_{0}, y_{0} ; \phi}$ can be found by means of the perturbation method for ordinary differential equations. Namely, let $\Lambda(t)=\mathbf{f}^{\prime}\left(\mathbf{x}_{\phi}(t)\right)$, where $\mathbf{f}=\left[\begin{array}{l}f_{1} \\ f_{2}\end{array}\right]$ and $\mathbf{f}^{\prime}$ is its Jacobian. Let $Q\left(t, t_{0}\right)$, for $T \geq t \geq t_{0} \geq 0$, be a matrix function such that $Q\left(t_{0}, t_{0}\right)=I, \partial Q\left(t, t_{0}\right) / \partial t=$ $\Lambda(t) Q\left(t, t_{0}\right)$ and $\mathbf{v}=\left[\begin{array}{l}\rho_{1} \\ \rho_{2}\end{array}\right]$. Then

$$
D_{x_{0}, y_{0} ; \phi} h=\int_{0}^{T} Q(T, s) \mathbf{v} h(s) d s .
$$

Lemma 4. Assume that $\rho_{1}, \rho_{2}>0, \tilde{a}_{1}, \tilde{a}_{2}>0$ and $\tilde{a}_{2} \rho_{1}>\tilde{a}_{1} \rho_{2}$. Let $M_{0}=\log \left(\left(b_{2}+c_{1}\right) /\left(b_{1}+c_{2}\right)\right)$ if $\rho_{2}=\rho_{1}$, and

$$
M_{0}=\log \left(\frac{\rho_{1}\left(b_{2} \rho_{1}+c_{1} \rho_{2}\right)}{\rho_{2}\left(b_{1} \rho_{2}+c_{2} \rho_{1}\right)}\left(\frac{\rho_{2}\left(\tilde{a}_{2} \rho_{1}-\tilde{a}_{1} \rho_{2}\right)}{\left(\rho_{1}-\rho_{2}\right)\left(b_{2} \rho_{1}+c_{1} \rho_{2}\right)}\right)^{1-\rho_{2} / \rho_{1}}\right)
$$

if $\rho_{2}<\rho_{1}$. Let $E=\mathbb{R}^{2}$ when $\rho_{2}>\rho_{1}$, and $E=E\left(M_{0}\right)$ when $\rho_{2} \leq \rho_{1}$, where

$$
E\left(M_{0}\right)=\left\{(x, y) \in \mathbb{R}^{2}: y>\left(\rho_{2} / \rho_{1}\right) x+M_{0}\right\} .
$$

Then for each $\left(x_{0}, y_{0}\right) \in E$ and for almost every $(x, y) \in E$ there exists $T>0$ such that $k\left(T, x, y ; x_{0}, y_{0}\right)>0$.

Proof. First, we show that there exists a constant $C$ such that the rank of $D_{x_{0}, y_{0}, \phi}$ is 2 if $y \neq x+C$, where $x=x_{\phi}(T)$ and $y=y_{\phi}(T)$. Let $\varepsilon \in(0, T)$ and $h_{\varepsilon}=1_{[T-\varepsilon, T]}$. Since $Q(T, s)=I+\Lambda(T)(T-s)+o(T-s)$, from 26) we obtain

$$
D_{x_{0}, y_{0} ; \phi} h_{\varepsilon}=\varepsilon \mathbf{v}+\frac{1}{2} \varepsilon^{2} \Lambda(T) \mathbf{v}+o\left(\varepsilon^{2}\right) .
$$

Since $\mathbf{v}=\left[\rho_{1}, \rho_{2}\right]$ and $\Lambda(T) \mathbf{v}=e^{x}\left[-c_{1} \rho_{1}+b_{1} \rho_{2} e^{y-x}, b_{2} \rho_{1}-c_{2} \rho_{2} e^{y-x}\right]$ there exists a constant

$$
C=\log \frac{\rho_{1}\left(b_{2} \rho_{1}+c_{1} \rho_{2}\right)}{\rho_{2}\left(b_{1} \rho_{2}+c_{2} \rho_{1}\right)}
$$

such that the vectors $\mathbf{v}$ and $\Lambda(T) \mathbf{v}$ are linearly independent if $y-x \neq C$. Consequently, $D_{x_{0}, y_{0} ; \phi}$ has rank 2 . 
Next we substitute

$$
z_{\phi}(t)=y_{\phi}(t)-\frac{\rho_{2}}{\rho_{1}} x_{\phi}(t)
$$

and replace system (24), (25) by

$$
\begin{aligned}
x_{\phi}^{\prime} & =\rho_{1} \phi+g_{1}\left(x_{\phi}, z_{\phi}\right), \\
z_{\phi}^{\prime} & =g_{2}\left(x_{\phi}, z_{\phi}\right)
\end{aligned}
$$

where

$$
g_{1}(x, z)=\tilde{a}_{1}-c_{1} e^{x}+b_{1} e^{z} e^{r x}, \quad g_{2}(x, z)=\alpha e^{x}-\beta e^{z} e^{r x}+\gamma
$$

with $\alpha=b_{2}+r c_{1}>0, \beta=c_{2}+r b_{1}>0, \gamma=\tilde{a}_{2}-r \tilde{a}_{1}>0, r=\rho_{2} / \rho_{1}$.

Now we check that for any two points $\left(x_{0}, z_{0}\right),\left(x_{1}, z_{1}\right) \in E$ there exist a control function $\phi$ and $T>0$ such that $x_{\phi}(0)=x_{0}, z_{\phi}(0)=z_{0}, x_{\phi}(T)=x_{1}$ and $z_{\phi}(T)=z_{1}$. The proof is split into the following cases.

$1^{\circ}$ Suppose $r>1$ and $E=\mathbb{R}^{2}$. First we find a positive constant $T$ and a differentiable function $z_{\phi}:[0, T] \rightarrow \mathbb{R}$ such that $z_{\phi}(0)=z_{0}, z_{\phi}(T)=z_{1}$,

$$
\alpha e^{x_{0}}-\beta e^{z_{0}} e^{r x_{0}}=z_{\phi}^{\prime}(0)-\gamma, \quad \alpha e^{x_{1}}-\beta e^{z_{1}} e^{r x_{1}}=z_{\phi}^{\prime}(T)-\gamma
$$

and

$$
z_{\phi}^{\prime}(t)-\gamma<\frac{\alpha(r-1)}{r}\left(\frac{\alpha}{r \beta e^{z_{\phi}(t)}}\right)^{1 /(r-1)} \quad \text { for } t \in[0, T] .
$$

In order to do it, we first determine $z_{\phi}^{\prime}(0)=: a_{0}$ and $z_{\phi}^{\prime}(T)=: a_{T}$ from (31). We construct the function $z_{\phi}$ separately in the three intervals $[0, \varepsilon],[\varepsilon, T-\varepsilon]$ and $[T-\varepsilon, T]$, where $0<\varepsilon<T / 2$. Since $\gamma>0, r>1$ and

$$
z_{\phi}^{\prime}(0)-\gamma<\frac{\alpha(r-1)}{r}\left(\frac{\alpha}{r \beta e^{z_{0}}}\right)^{1 /(r-1)}
$$

we can construct a $C^{2}$ function $z_{\phi}:[0, \varepsilon] \rightarrow \mathbb{R}$ such that $z_{\phi}(0)=z_{0}, z_{\phi}^{\prime}(0)$ $=a_{0}, z_{\phi}^{\prime}(\varepsilon)=0$ and $z_{\phi}$ satisfies inequality $(32)$ for $t \in[0, \varepsilon]$. In the same way we construct $z_{\phi}:[T-\varepsilon, T] \rightarrow \mathbb{R}$ such that $z_{\phi}(T)=z_{1}, z_{\phi}^{\prime}(T)=a_{T}$, $z_{\phi}^{\prime}(T-\varepsilon)=0$ and $z_{\phi}$ satisfies $(32)$ for $t \in[T-\varepsilon, T]$. Taking sufficiently large $T$ we can extend $z_{\phi}:[0, \varepsilon] \cup[T-\varepsilon, T] \rightarrow \mathbb{R}$ to a $C^{2}$ function defined on the whole interval $[0, T]$ such that $z_{\phi}^{\prime}(t)<\gamma$ for $t \in[\varepsilon, T-\varepsilon]$, and therefore $z_{\phi}$ satisfies (32). From (32) it follows that we can find a $C^{1}$ function $x_{\phi}$ which satisfies equation (29) and finally we can determine a continuous function $\phi$ from $(28)$.

$2^{\circ}$ Assume that $r=1$. Thus $M_{0}=\log (\alpha / \beta)$. Let

$$
E=E\left(M_{0}\right)=\left\{(x, z) \in \mathbb{R}^{2}: z>M_{0}\right\} .
$$

In this case we construct the function $\phi$ in the following way. First we find a positive constant $T$ and a differentiable function $z_{\phi}:[0, T] \rightarrow \mathbb{R}$ such that 
$z_{\phi}(0)=z_{0}, z_{\phi}(T)=z_{1}$,

$$
e^{x_{0}}\left(\alpha-\beta e^{z_{0}}\right)=z_{\phi}^{\prime}(0)-\gamma, \quad e^{x_{1}}\left(\alpha-\beta e^{z_{1}}\right)=z_{\phi}^{\prime}(T)-\gamma
$$

and

$$
z_{\phi}^{\prime}(t)-\gamma<0, \quad \alpha-\beta e^{z(t)}<0 \quad \text { for } t \in[0, T] .
$$

In order to do it, we first determine $z_{\phi}^{\prime}(0)=: a_{0}$ and $z_{\phi}^{\prime}(T)=: a_{T}$ from 33 . Next let $0<\varepsilon<T / 2$. Since $\gamma>0$, $z_{0}>M_{0}$ and $z_{\phi}^{\prime}(0)-\gamma<0$ we can construct a $C^{2}$ function $z_{\phi}:[0, \varepsilon] \rightarrow \mathbb{R}$ such that $z_{\phi}(0)=z_{0}, z_{\phi}^{\prime}(0)=a_{0}$, $z_{\phi}^{\prime}(\varepsilon)=0$ and $z_{\phi}$ satisfies (34) for $t \in[0, \varepsilon]$. In the same way we construct $z_{\phi}:[T-\varepsilon, T] \rightarrow \mathbb{R}$ such that $z_{\phi}(T)=z_{1}, z_{\phi}^{\prime}(T)=a_{T}, z_{\phi}^{\prime}(T-\varepsilon)=0$ and $z_{\phi}$ satisfies (34) for $t \in[T-\varepsilon, T]$. Taking sufficiently large $T$ we can extend $z_{\phi}:[0, \varepsilon] \cup[T-\varepsilon, T] \rightarrow \mathbb{R}$ to a $C^{2}$ function defined on $[0, T]$ such that (34) holds. From (34) it follows that we can find a $C^{1}$ function $x_{\phi}$ which satisfies (29) and finally we determine a continuous function $\phi$ from 28.

$3^{\circ}$ Assume that $r \in(0,1)$ and $E=E\left(M_{0}\right)=\left\{(x, z) \in \mathbb{R}^{2}: z>M_{0}\right\}$, where

$$
M_{0}=\log \left(\frac{\alpha}{r \beta}\left(\frac{r \gamma}{\alpha(1-r)}\right)^{1-r}\right) .
$$

In this case we construct the function $\phi$ in the following way. First, we find a positive constant $T$ and a differentiable function $z_{\phi}:[0, T] \rightarrow \mathbb{R}$ such that $z_{\phi}(0)=z_{0}, z_{\phi}(T)=z_{1}$,

$$
\alpha e^{x_{0}}-\beta e^{z_{0}} e^{r x_{0}}=z_{\phi}^{\prime}(0)-\gamma, \quad \alpha e^{x_{1}}-\beta e^{z_{1}} e^{r x_{1}}=z_{\phi}^{\prime}(T)-\gamma,
$$

and

$$
\begin{aligned}
z_{\phi}^{\prime}(t)-\gamma<\frac{\alpha(r-1)}{r}\left(\frac{\alpha}{r \beta e^{z_{\phi}(t)}}\right)^{1 /(r-1)} & \text { for } t \in[0, T], \\
\frac{\alpha(r-1)}{r}\left(\frac{\alpha}{r \beta e^{z_{\phi}(t)}}\right)^{1 /(r-1)}+\gamma>0 & \text { for } t \in[0, T] .
\end{aligned}
$$

In order to do it, we first determine $z_{\phi}^{\prime}(0)=: a_{0}$ and $z_{\phi}^{\prime}(T)=: a_{T}$ from (35). Next let $0<\varepsilon<T / 2$. Since $\gamma>0, z_{0}>M_{0}$ and

$$
z_{\phi}^{\prime}(0)-\gamma<\frac{\alpha(r-1)}{r}\left(\frac{\alpha}{r \beta e^{z_{0}}}\right)^{1 /(r-1)}
$$

we can construct a $C^{2}$ function $z_{\phi}:[0, \varepsilon] \rightarrow \mathbb{R}$ such that $z_{\phi}(0)=z_{0}, z_{\phi}^{\prime}(0)$ $=a_{0}, z_{\phi}^{\prime}(\varepsilon)=0$ and $z_{\phi}$ satisfies (36) and (37) for $t \in[0, \varepsilon]$. Analogously, we construct $z_{\phi}:[T-\varepsilon, T] \rightarrow \mathbb{R}$ such that $z_{\phi}(T)=z_{1}, z_{\phi}^{\prime}(T)=a_{T}, z_{\phi}^{\prime}(T-\varepsilon)=0$ and $z_{\phi}$ satisfies (36) and (37) for $t \in[T-\varepsilon, T]$. Taking sufficiently large $T$ we can extend $z_{\phi}:[0, \varepsilon] \cup[T-\varepsilon, T] \rightarrow \mathbb{R}$ to a $C^{2}$ function defined on $[0, T]$ such that (36) and (37) hold. Hence we can find a $C^{1}$ function $x_{\phi}$ which satisfies 29) and finally we can determine a continuous function $\phi$ from 28 . 
From cases $1^{\circ}-3^{\circ}$ it follows that for any $\left(x_{0}, y_{0}\right),(x, y) \in E$ there is a control function $\phi$ and $T>0$ such that $x_{\phi}(0)=x_{0}, y_{\phi}(0)=y_{0}, x_{\phi}(T)=x$ and $y_{\phi}(T)=y$. From the first part of the proof we now conclude that $k\left(T, x, y ; x_{0}, y_{0}\right)>0$ if $y \neq x+C$.

From Lemma 4 we obtain the following results.

Lemma 5. Assume that $\rho_{1}, \rho_{2}>0, \tilde{a}_{1}, \tilde{a}_{2}>0$ and $\tilde{a}_{2} \rho_{1}<\tilde{a}_{1} \rho_{2}$. Let $M_{0}=\log \left(\left(b_{2}+c_{1}\right) /\left(b_{1}+c_{2}\right)\right)$ if $\rho_{2}=\rho_{1}$, and

$$
M_{0}=\log \left(\frac{\rho_{1}\left(b_{2} \rho_{1}+c_{1} \rho_{2}\right)}{\rho_{2}\left(b_{1} \rho_{2}+c_{2} \rho_{1}\right)}\left(\frac{\rho_{2}\left(\tilde{a}_{2} \rho_{1}-\tilde{a}_{1} \rho_{2}\right)}{\left(\rho_{1}-\rho_{2}\right)\left(b_{2} \rho_{1}+c_{1} \rho_{2}\right)}\right)^{1-\rho_{2} / \rho_{1}}\right)
$$

if $\rho_{2}>\rho_{1}$. Let $E=\mathbb{R}^{2}$ when $\rho_{2}<\rho_{1}$ and $E=E\left(M_{0}\right)$ when $\rho_{2} \geq \rho_{1}$, where

$$
E\left(M_{0}\right)=\left\{(x, y) \in \mathbb{R}^{2}: y<\left(\rho_{2} / \rho_{1}\right) x+M_{0}\right\} .
$$

Then for each $\left(x_{0}, y_{0}\right) \in E$ and for almost every $(x, y) \in E$ there exists $T>0$ such that $k\left(T, x, y ; x_{0}, y_{0}\right)>0$.

Lemma 6. Assume that $\rho_{1}, \rho_{2}>0, \tilde{a}_{1}>0$ and $\tilde{a}_{2}<0$. Let $M_{0}=$ $\log \left(\left(b_{2}+c_{1}\right) /\left(b_{1}+c_{2}\right)\right)$ if $\rho_{2}=\rho_{1}$ and

$$
M_{0}=\log \left(\frac{\rho_{1}\left(b_{2} \rho_{1}+c_{1} \rho_{2}\right)}{\rho_{2}\left(b_{1} \rho_{2}+c_{2} \rho_{1}\right)}\left(\frac{\rho_{2}\left(\tilde{a}_{2} \rho_{1}-\tilde{a}_{1} \rho_{2}\right)}{\left(\rho_{1}-\rho_{2}\right)\left(b_{2} \rho_{1}+c_{1} \rho_{2}\right)}\right)^{1-\rho_{2} / \rho_{1}}\right)
$$

if $\rho_{2}>\rho_{1}$. Let $E=\mathbb{R}^{2}$ when $\rho_{2}<\rho_{1}$ and $E=E\left(M_{0}\right)$ when $\rho_{2} \geq \rho_{1}$, where

$$
E\left(M_{0}\right)=\left\{(x, y) \in \mathbb{R}^{2}: y<\left(\rho_{2} / \rho_{1}\right) x+M_{0}\right\} .
$$

Then for each $\left(x_{0}, y_{0}\right) \in E$ and for almost every $(x, y) \in E$ there exists $T>0$ such that $k\left(T, x, y ; x_{0}, y_{0}\right)>0$.

In case (ii) when only the second population is stochastically perturbed we have

Lemma 7. Assume that $\rho_{1}=0, \rho_{2}>0$. Let $E=\left(\log \left(a_{1} / c_{1}\right), \infty\right) \times \mathbb{R}$. Then for each $\left(x_{0}, y_{0}\right) \in E$ and for almost every $(x, y) \in E$ there exists $T>0$ such that $k\left(T, x, y ; x_{0}, y_{0}\right)>0$.

Proof. First, we show that the rank of $D_{x_{0}, y_{0}, \phi}$ is 2 . Let $\varepsilon \in(0, T)$ and $h_{\varepsilon}=1_{[T-\varepsilon, T]}$. Since the vectors $\mathbf{v}=\left[0, \rho_{2}\right]$ and $\Lambda(T) \mathbf{v}=e^{y} \rho_{2}\left[b_{1},-c_{2}\right]$ are linearly independent, from (27) it follows that $D_{x_{0}, y_{0} ; \phi}$ has rank 2 .

Next we prove that for any $\left(x_{0}, y_{0}\right),\left(x_{1}, y_{1}\right) \in E$ there exist a control function $\phi$ and $T>0$ such that $x_{\phi}(0)=x_{0}, y_{\phi}(0)=y_{0}, x_{\phi}(T)=x_{1}$ and $y_{\phi}(T)=y_{1}$. First we find a positive constant $T$ and a differentiable function $x_{\phi}:[0, T] \rightarrow \mathbb{R}$ such that $x_{\phi}(0)=x_{0}, x_{\phi}(T)=x_{1}$,

$$
b_{1} e^{y_{0}}=x_{\phi}^{\prime}(0)-a_{1}+c_{1} e^{x_{0}}, \quad b_{1} e^{y_{1}}=x_{\phi}^{\prime}(T)-a_{1}+c_{1} e^{x_{1}}
$$

and

$$
x_{\phi}^{\prime}(t)-a_{1}+c_{1} e^{x_{\phi}(t)}>0 \quad \text { and } \quad a_{1}-c_{1} e^{x_{\phi}(t)}<0 \quad \text { for } t \in[0, T] .
$$


In order to do it, we first determine $x_{\phi}^{\prime}(0)=: a_{0}$ and $x_{\phi}^{\prime}(T)=: a_{T}$ from (38). Next let $0<\varepsilon<T / 2$. Since $c_{1} e^{x_{0}}>a_{1}$ and $x_{\phi}^{\prime}(0)-a_{1}+c_{1} e^{x_{0}}>0$ we can construct a $C^{2}$ function $x_{\phi}:[0, \varepsilon] \rightarrow \mathbb{R}$ such that $x_{\phi}(0)=x_{0}, x_{\phi}^{\prime}(0)=a_{0}$, $x_{\phi}^{\prime}(\varepsilon)=0$ and $x_{\phi}$ satisfies $[39)$ for $t \in[0, \varepsilon]$. In the same way we construct $x_{\phi}:[T-\varepsilon, T] \rightarrow \mathbb{R}$ such that $x_{\phi}(T)=x_{1}, x_{\phi}^{\prime}(T)=a_{T}, x_{\phi}^{\prime}(T-\varepsilon)=0$ and $x_{\phi}$ satisfies (39) for $t \in[T-\varepsilon, T]$. Taking sufficiently large $T$ we can extend $x_{\phi}:[0, \varepsilon] \cup[T-\varepsilon, T] \rightarrow \mathbb{R}$ to a $C^{2}$ function defined on $[0, T]$ such that (39) holds. It follows that we can find a $C^{1}$ function $y_{\phi}$ which satisfies (24) and finally we can determine a continuous function $\phi$ from (25).

Lemma 8. Assume that $\rho_{1}, \rho_{2}>0, \tilde{a}_{1}, \tilde{a}_{2}>0, \tilde{a}_{2} \rho_{1}>\tilde{a}_{1} \rho_{2}$, and $\rho_{2} \leq \rho_{1}$. Let $E=E\left(M_{0}\right)$. Then for every density $f$ we have

$$
\lim _{t \rightarrow \infty} \iint_{E} P(t) f(x) d x d y=1
$$

Proof. First we substitute

$$
\zeta(t)=\eta(t)-\frac{\rho_{2}}{\rho_{1}} \xi(t) .
$$

Then we replace system (15), (16) by

$$
\begin{aligned}
& d \xi(t)=g_{1}(\xi(t), \zeta(t)) d t+\rho_{1} d W(t), \\
& d \zeta(t)=g_{2}(\xi(t), \zeta(t)) d t,
\end{aligned}
$$

where the functions $g_{1}, g_{2}$ are defined by (30). Since for each $\varepsilon>0$ we have

$$
\inf \left\{g_{2}(x, z): z \leq M_{0}-\varepsilon, x \in \mathbb{R}\right\}>0
$$

we obtain $\liminf \operatorname{in}_{t \rightarrow \infty} \zeta_{t} \geq M_{0}$. We will prove that for almost every $\omega$ there is $t_{0}=t_{0}(\omega)$ such that $\zeta(t, \omega)>M_{0}$ for $t \geq t_{0}$.

The case $\rho_{2}=\rho_{1}$ is obvious, because $g_{2}\left(x, M_{0}\right)=\gamma>0$ for all $x \in \mathbb{R}$. Consider the case $\rho_{2}<\rho_{1}$. Thus there exists $C_{0} \in \mathbb{R}$ such that $g_{2}\left(C_{0}, M_{0}\right)$ $=0$. Fix $\kappa>0$ and $\tau>0$. Consider the solution of system 41, 42 with initial conditions $\xi(0)=C_{0}+2 \kappa, \zeta(0)=M_{0}-\tau$. Let

$A_{\kappa, \tau}=\left[C_{0}, C_{0}+\kappa\right] \times\left[M_{0}-\tau, M_{0}\right], \quad B_{\kappa, \tau}=\left[C_{0}, C_{0}+2 \kappa\right] \times\left[M_{0}-\tau, M_{0}\right]$.

Then from the continuity of $g_{1}, g_{2}$ it follows that there exist $\varepsilon, L>0$ such that $g_{2}(x, z)>\varepsilon$ for $x \geq C_{0}+\kappa, z \in\left[M_{0}-\tau, M_{0}\right]$ and $\left|g_{1}(x, z)\right| \leq L$ for $(x, z) \in B_{\kappa, \tau}$. Let $\bar{\xi}(t)$ be a solution of the equation $d \bar{\xi}(t)=-L d t+\rho_{1} d W(t)$ such that $\bar{\xi}(0)=C_{0}+2 \kappa$. From the comparison theorem we obtain $\bar{\xi}(t) \leq \xi(t)$ and $\zeta(t)>M_{0}-\tau+\varepsilon t$ as long as $(\xi(t), \zeta(t)) \in B_{\kappa, \tau} \backslash A_{\kappa, \tau}$. Let $t=\tau / \varepsilon$ and $\Omega_{\tau}=\left\{\omega: \bar{\xi}(s, \omega) \geq C_{0}+\kappa\right.$ for $\left.s \leq t\right\}$. Thus $\lim _{\tau \rightarrow 0} \operatorname{Prob}\left(\Omega_{\tau}\right)=1$ and $\zeta(t, \omega)>M_{0}$ for $\omega \in \Omega_{\tau}$. Now let $(\xi(t), \zeta(t))$ be any solution of system 41, (42). Then from what has already been proved and from the Markov property it follows that if $\sup _{t>0} \zeta(t, \omega) \leq M_{0}$ then $\lim \sup _{t \rightarrow \infty} \xi(t, \omega) \leq C_{0}$. In the same way we check that if $\sup _{t>0} \zeta(t, \omega) \leq M_{0}$ then $\liminf _{t \rightarrow \infty} \xi(t, \omega)$ 
$\geq C_{0}$. Thus $\sup _{t>0} \zeta(t, \omega) \leq M_{0}$ implies $\lim _{t \rightarrow \infty} \xi(t, \omega)=C_{0}$. Assume that $\lim _{t \rightarrow \infty} \xi(t, \omega)=C_{0}$ with probability $>p_{0}>0$. Let $\gamma_{1}=g_{1}\left(C_{0}, M_{0}\right)$. Then for every $\varepsilon>0$ there exist $t_{0}>0$ and a set $\Omega^{\prime}$ such that $\operatorname{Prob}\left(\Omega^{\prime}\right)>p_{0}$, $\left|\xi(t, \omega)-C_{0}\right|<\varepsilon$ and

$$
\rho_{1} d W(t)+\left(\gamma_{1}-\varepsilon\right) d t \leq d \xi(t) \leq \rho_{1} d W(t)+\left(\gamma_{1}+\varepsilon\right) d t
$$

for $\omega \in \Omega^{\prime}$ and $t \geq t_{0}$. Then $\operatorname{Prob}\left(\left\{\omega \in \Omega^{\prime}:\left|\xi\left(t_{0}+1\right)-C_{0}\right|<\varepsilon\right\}\right) \leq O(\varepsilon)$, which contradicts the assumption that $p_{0}>0$. Consequently, for almost every $\omega$ there exists $t_{0}=t_{0}(\omega)$ such that $\zeta(t, \omega)>M_{0}$ for $t \geq t_{0}$ and (40) holds.

From Lemma 8 we obtain the following results.

Lemma 9. Assume that $\rho_{1}, \rho_{2}>0, \tilde{a}_{1}, \tilde{a}_{2}>0, \tilde{a}_{2} \rho_{1}<\tilde{a}_{1} \rho_{2}$, and $\rho_{2} \geq \rho_{1}$. Let $E=E\left(M_{0}\right)$. Then for every density $f$ we have

$$
\lim _{t \rightarrow \infty} \iint_{E} P(t) f(x) d x d y=1 .
$$

Lemma 10. Assume that $\rho_{1}, \rho_{2}>0, \tilde{a}_{1}>0, \tilde{a}_{2}<0$, and $\rho_{2} \geq \rho_{1}$. Let $E=E\left(M_{0}\right)$. Then for every density $f$ we have

$$
\lim _{t \rightarrow \infty} \iint_{E} P(t) f(x) d x d y=1 .
$$

LEMma 11. Consider case (ii) when only the second population is stochastically perturbed. Assume that either $\tilde{a}_{2}>0$, or $\tilde{a}_{2}<0$ and $a_{1} b_{2}+\tilde{a}_{2} c_{1}>0$. Let $E=\left(\log \left(a_{1} / c_{1}\right), \infty\right) \times \mathbb{R}$. Then for every density $f$ we have

$$
\lim _{t \rightarrow \infty} \iint_{E} P(t) f(x) d x d y=1 .
$$

Proof. The process $\xi(t)$ satisfies the differential equation

$$
d \xi(t)=\left(a_{1}+b_{1} e^{\eta(t)}-c_{1} e^{\xi(t)}\right) d t .
$$

It follows that for every $\omega$ there are two cases:

(a) there is $t_{0}=t_{0}(\omega)$ such that $\xi(t, \omega)>\log \left(a_{1} / c_{1}\right)$ for $t>t_{0}$,

(b) $\lim _{t \rightarrow \infty} \xi(t, \omega)=\log \left(a_{1} / c_{1}\right)$ and $\lim _{t \rightarrow \infty} \eta(t, \omega)=-\infty$.

Case (b) is impossible because from the assumption $a_{1} b_{2}+\tilde{a}_{2} c_{1}>0$ and the equation

$$
d \eta(t)=\left(\tilde{a}_{2}+b_{2} e^{\xi(t)}-c_{2} e^{\eta(t)}\right) d t+\rho_{2} d W(t)
$$

it follows that there exists $\varepsilon>0$ such that

$$
d \eta(t) \geq \varepsilon d t+\rho_{2} d W(t)
$$

for sufficiently large $t$, which contradicts the fact that $\lim _{t \rightarrow \infty} \eta(t, \omega)=$ $-\infty$. 
TheOREM 7. Let $b_{1} b_{2}<c_{1} c_{2}$. In case (i) assume that $\rho_{1} \neq \rho_{2}$ or $\tilde{a}_{1} \neq \tilde{a}_{2}$. If either $\tilde{a}_{1}, \tilde{a}_{2}>0$, or $\tilde{a}_{1}>0, \tilde{a}_{2}<0$ and $\tilde{a}_{1} b_{2}+\tilde{a}_{2} c_{1}>0$, then the semigroup $\{P(t)\}_{t \geq 0}$ is asymptotically stable.

Proof. From Lemmas 811 it follows that it is sufficient to consider the restriction of the semigroup $\{P(t)\}_{t \geq 0}$ on $L^{1}\left(\mathbb{R}^{2}\right)$ to the space $L^{1}(E)$. From Lemmas 2, 3 we can see that $\{P(t)\}_{t \geq 0}$ is an integral Markov semigroup with a continuous kernel. According to Lemmas 47 we have

$$
\int_{0}^{\infty} P(t) f d t>0 \quad \text { a.e. on } E
$$

for every $f \in D$. From Theorem 4 we conclude that $\{P(t)\}_{t \geq 0}$ satisfies the Foguel alternative. In order to exclude sweeping we construct a Khasminskiu function exactly as in [28]. Thus the semigroup $\{P(t)\}_{t \geq 0}$ is asymptotically stable.

\section{References}

[1] S. Aida, S. Kusuoka and D. Stroock, On the support of Wiener functionals, in: Asymptotic Problems in Probability Theory: Wiener Functionals and Asymptotic, K. D. Elworthy and N. Ikeda (eds.), Pitman Res. Notes Math. Ser. 284, Longman Sci. Tech., 1993, 3-34.

[2] F. Baudoin, An Introduction to the Geometry of Stochastic Flows, Imperial College Press, London, 2004.

[3] G. Ben Arous et R. Léandre, Décroissance exponentielle du noyau de la chaleur sur la diagonale (II), Probab. Theory Related Fields 90 (1991), 377-402.

[4] D. H. Boucher, The Biology of Mutualism, Oxford Univ. Press, 1988.

[5] S. Chessa and H. Fujita Yashima, The stochastic equation of predator-prey population dynamics, Boll. Un. Mat. Ital. Sez. B (8) 5 (2002), 789-804 (in Italian).

[6] A. Friedman, Stochastic Differential Equations and Applications, Vol. 2, Academic Press, New York, 1976.

[7] G. F. Gause and A. A. Witt, Behaviour of mixed populations and the problem of natural selection, Amer. Naturalist 69 (1935), 596-609.

[8] I. I. Gihman and A. V. Skorohod, Stochastic Differential Equations, Springer, Berlin, 1972.

[9] L. Hörmander, Hypoelliptic second order differential equations, Acta Math. 119 (1967), 147-171.

[10] R. Z. Khasminski1̌, Ergodic properties of recurrent diffusion processes and stabilization of the solution to the Cauchy problem for parabolic equations, Theory Probab. Appl. 5 (1960), 179-195.

[11] _, Stochastic Stability of Differential Equations, Sijthoff \& Noordhoff, Alphen aan den Rijn, 1980.

[12] V. A. Kostitzin, Symbiose, parasitisme et évolution, Hermann, Paris, 1934.

[13] H. Kunita, Supports of diffusion processes and controllability problems, in: Proc. Int. Symp. SDE (Kyoto, 1976), Wiley, 1978, 163-185.

[14] A. Lasota and M. C. Mackey, Chaos, Fractals and Noise. Stochastic Aspects of Dynamics, Appl. Math. Sci. 97, Springer, New York, 1994. 
[15] P. Malliavin, Stochastic calculus of variations and hypoelliptic operators, in: Proc. Int. Symp. SDE (Kyoto, 1976), Wiley, 1978, 195-263.

[16],$- C^{k}$-hypoellipticity with degeneracy, in: Stochastic Analysis, Academic Press, New York, 1978, 199-214.

[17] J. D. Murray, Mathematical Biology. I: An Introduction, Springer, New York, 2002.

[18] K. Narita, No explosion criteria for stochastic differential equations, J. Math. Soc. Japan 34 (1982), 191-203.

[19] J. Norris, Simplified Malliavin calculus, in: Séminaire de probabilités XX, Lecture Notes in Math. 1204, Springer, 1986, 101-130.

[20] K. Pichór and R. Rudnicki, Stability of Markov semigroups and applications to parabolic systems, J. Math. Anal. Appl. 215 (1997), 56-74.

[21] —, - Asymptotic behaviour of Markov semigroups and applications to transport equations, Bull. Polish Acad. Sci. Math. 45 (1997), 279-397.

[22] - - - Continuous Markov semigroups and stability of transport equations, J. Math. Anal. Appl. 249 (2000), 668-685.

[23] A. Rescigno and W. Richardson, The struggle for life I: Two species, Bull. Math. Biophys. 29 (1967), 377-388.

[24] R. Rudnicki, On asymptotic stability and sweeping for Markov operators, Bull. Polish Acad. Sci. Math. 43 (1995), 245-262.

[25] —, Long-time behaviour of a stochastic prey-predator model, Stoch. Process. Appl. 108 (2003), 93-107.

[26] R. Rudnicki and K. Pichór, Influence of stochastic perturbation on prey-predator systems, Math. Biosci. 206 (2007), 108-119.

[27] R. Rudnicki, K. Pichór and M. Tyran-Kamińska, Markov semigroups and their applications, Markov semigroups and their applications, in: Dynamics of Dissipation, P. Garbaczewski and R. Olkiewicz (eds.), Lecture Notes in Phys. 597, Springer, Berlin, 215-238.

[28] U. Skwara, A stochastic model of symbiosis, Ann. Polon. Math. 97 (2010), 257-272.

[29] D. W. Stroock and S. R. S. Varadhan, On the support of diffusion processes with applications to the strong maximum principle, in: Proc. Sixth Berkeley Symposium on Mathematical Statistics and Probability, Vol. III, Univ. Calif. Press, Berkeley, 1972, 333-360.

[30] E. Tornatore, L. Manca and H. Fujita Yashima, Comportamento asintotico della soluzione del sistema di equazioni stocastiche per due specie in competizione, Istit. Lombardo Rend. A 136-137 (2004), 151-183.

Urszula Skwara

Institute of Mathematics

Maria Curie-Skłodowska University

Pl. M. Curie-Skłodowskiej 1

20-031 Lublin, Poland

E-mail: uskwara@umcs.lublin.pl

uskwara@o2.pl

Received 28.5.2009

and in final form 25.9.2009 